\title{
RADIOGRAPHIC ASSESSMENT OF IMPACTED MAXILLARY CANINE POSITION USING CBCT: A COMPARATIVE STUDY OF 2 METHODS
}

\author{
Dina M. El Beshlawy* and Dina F. Ahmed ${ }^{* *}$
}

\begin{abstract}
Objectives: The aim of this study was to compare and evaluate the guidelines proposed by Counihan et al 2013 and the KPG index method for assessment of maxillary impacted canine position using CBCT images from a radiographic point of view.

Subjects \& methods: Thirty-six CBCT scans of 36 patients (6 males and 30 females), with a total number of 45 impacted maxillary canines, were recruited from the database of the Oral and Maxillofacial Radiology Department, Faculty of Dentistry, Cairo University. KPG index, for each impacted canine, was calculated by giving both its cusp and root tips a score on a $0-5$ scale along the $x, y$, and $z$ planes. The summation of these six scores would predict the treatment difficulty, classified as easy (0-9), moderate (10-14), difficult (15-19), and extremely difficult (20 and above). Moreover, Counihan et al 2013 guidelines were applied on each studied impacted tooth in which the tooth position in four categories was considered: overlap with adjacent incisor, vertical height, angulation to midline and position of root apex. For each category, either good, average or poor prognostic outcome was selected. Data were collected and statistically analyzed.
\end{abstract}

Results Upon comparing both studied methods of radiographic assessment of impacted maxillary canines, a statistically significant difference was found: KPG index categorized more cases (9 cases) as easy (20\%) while Counihan et al guidelines categorized only 2 cases $(4.4 \%)$ as having good prognosis. Similarly, KPG index categorized more cases (15 cases) as moderate $(33.3 \%)$ while Counihan et al guidelines categorized 13 cases $(28.9 \%)$ as having average prognosis. On the other hand, Counihan et al guidelines categorized more cases (30 cases) as poor prognosis $(66.7 \%)$ while KPG index categorized only 21 cases $(46.7 \%)$ as being difficult. Nonetheless, on comparing the treatment difficulty and prognosis of impacted maxillary canines found on the right and left sides, unilaterally or bilaterally, in males and females, a non-statistically significant difference was found using both methods of radiographic assessment.

Conclusion From a radiographic point of view, the KPG index, with some minute modifications, has demonstrated to be an effective and competent method for classification of maxillary impacted canine's treatment difficulty in accordance to their position, as compared to the guidelines proposed by Counihan et al at 2013 .

KEY WORDS: CBCT, impacted canine, KPG index, guidelines.

*Associate Professor of Oral and Maxillofacial Radiology, Faculty of Dentistry, Cairo University.

** Lecturer of Oral and Maxillofacial Radiology, Faculty of Dentistry, Cairo University. 


\section{INTRODUCTION}

An impacted tooth is defined as one that fails to erupt at its appropriate location in the dental arch, within its normal period of development ${ }^{1}$. Maxillary canines are the most frequently impacted teeth, after third molars, with a prevalence range of 0.9-3.0\%, according to the studied population ${ }^{2}$. Impaction of maxillary canines occurs commonly as they have a prolonged deep development period in the maxilla and a long pathway of eruption that may be complicated with the presence of other teeth ${ }^{3}$. Females seem to be more commonly affected than males, for an unknown etiology ${ }^{4}$.

Having a critical masticatory and esthetical role and being a corner stone of the dental occlusion and stability, it's important that clinicians are aware of any maxillary canines related pathological condition as early as possible. This, in turn, will allow a more accurate diagnosis and treatment planning thus preventing any of the possible complications associated with its incidence and/or management ${ }^{5,6}$.

Previously, conventional two-dimensional radiographic imaging modalities, especially panoramic radiographs, were most commonly used for diagnosis and treatment planning of the impacted maxillary canines. Still, some drawbacks such as distortion, magnification, blurring, and projection of the three-dimensional complex maxillofacial structures on a two-dimensional image could decrease the value of panoramic radiographs and rise the misinterpretation risks ${ }^{7}$. Accordingly, many studies recommended that the use of panoramic images alone are not satisfactory for formulation of a correct treatment plans for impacted maxillary canines $^{7,8}$.

Cone Beam Computed Tomography (CBCT), a relatively new imaging technique, offers undistorted three-dimensional images of dental structures with higher spatial resolution and lower dosages of radiation compared to conventional Computed Tomography (CT) scans ${ }^{4,9}$. From the volumetric data, various manufacturer softwares enable different reconstructions valuable for evaluation of impacted teeth and neighboring structures ${ }^{4}$.

Regarding the radiographic assessment of maxillary impacted canines, Counihan et al at $2013^{10}$ offered some guidelines in which the tooth position in four categories is considered: overlap with adjacent incisor, vertical height, angulation to midline and position of root apex. For each category, either good, average or poor prognostic outcome is selected. The evidence basics for these predictive indicators have been investigated in several previous studies ${ }^{11,12,13}$.

In 2009, KPG index, an innovative threedimensional classification system for assessment of maxillary canine impactions, was introduced. This index classifies impacted canines, relying on their distance from their normal position, by giving both its cusp and root tips a score on a $0-5$ scale along the $x, y$, and $z$ planes. The summation of these six scores would predict the treatment difficulty, classified as easy (0-9), moderate (10-14), difficult (15-19), and extremely difficult (20 and above). The authors of this index used the Sirona Galileos CBCT scanner for imaging of 42 impacted maxillary canines and evaluated the resultant images with the Galaxis software ${ }^{4}$.

The index validity was investigated in several studies: San Martin et al studied the ability of the KPG index to estimate the required treatment time of an impacted canine and found that its use with further verification will become more and more valuable to orthodontists ${ }^{14}$. Dalessandri et al investigated its inter- and intra-observer agreement and found that the reliability of the KPG index, with some additional practical recommendations, could reach a perfect agreement and that its reproducibility is not affected by the type of used CBCT scanner, especially when the voxel size and slice thickness are identical ${ }^{15}$. Kau et al evaluated the level of agreement of orthodontists in the management of 
impacted maxillary canines using the KPG index and concluded that the index based on the 3D coordinates of the spatial location of the canine may be merged into the clinical practice ${ }^{16}$.

The aim of this study was to compare and evaluate the guidelines proposed by Counihan et al 2013 and the KPG index method for assessment of maxillary impacted canine position using CBCT images from a radiographic point of view.

\section{SUBJECTS AND METHODS}

The study sample was recruited from the CBCT scans database of the Oral and Maxillofacial Radiology Department, Faculty of Dentistry, Cairo University, where the patients had been referred for CBCT examination either from the orthodontic and/or the surgery department for diagnosis and treatment plan formulation.

The scans were selected according to the following inclusion criteria: maxillary canine impaction (left or right, uni or bilateral); patient's age over 13 years (root closure is nearly completed), scans were either 0.2 or 0.4 voxel size. However, scans of patients with craniofacial syndromes or dental anomalies that could disturb tooth development or eruption, scans with artifacts that unable the assessment of the maxillary arch, scans with extremely positioned impacted canine away from the dental arch were excluded. Thirty-six CBCT scans of 36 patients (6 males and 30 females) with an age range varying from 13 to 35 years were included in the current study with a total number of 45 impacted maxillary canines.

CBCT scans were acquired by Planmeca ProMax 3D Mid scanner (Helsinki, Finland). All scans were taken with the following parameters: $90 \mathrm{kVp}, 8 \mathrm{~mA}$, 12 or $13.5 \mathrm{sec}$, FOV 500 x 500 and $0.2-0.4 \mathrm{~mm}$ voxel size. The acquired images, next to Digital Imaging and Communications in Medicine (DICOM) files export, were processed and analyzed with the Romexis Viewer 4.5.0.R on a personal computer running Microsoft Windows 10 (Microsoft Corp, Redmond, WA, USA). For standardization, all scans were analyzed after adjusting the slice thickness to $0.4 \mathrm{~mm}$.

\section{Image analysis}

All assessment procedures were performed once by two radiologists, with more than 15 years of experience in the field of Oral and Maxillofacial Radiology:

\section{KPG index:}

KPG index was calculated by addition of the cusp and root tips scores (from 0 to 5) on x (mesio-distal), y (vertical) and z (labio-lingual) planes. According to the original version scores, the range 0-9 fell into the category of easy, 10-14 were considered as moderate, 15-19 were taken as difficult while 2030 were extremely difficult. The same methodology was applied in many previous studies ${ }^{4,15,16}$.

First, on a selected CBCT axial cut, at the level of impacted maxillary canine, a panoramic curve was drawn. Using the reformatted panoramic view, with thickness increased until showing the impacted tooth relation with the adjacent teeth, the following were evaluated: The mesio-distal (x axis) and vertical (y axis) positions of both the cusp and root tips in relation to its normal developmental position were evaluated and given a score from 0 to 5 (figure 1).

Then, on another selected axial cut showing the cusp tip of the impacted maxillary canine, the labiolingual ( $\mathrm{z}$ axis) position was evaluated and given a score from 0 to 5 . This was based on the distance of the impacted tip to the occlusal reference arch in $2 \mathrm{~mm}$ increment (figure 1). For the labio-lingual ( $z$ axis) position of the root tip, the authors of the current study found a great difficulty in localization of the root tip as it was usually located at a higher position in relation to the occlusal reference arch. Thus, they suggested a simple method of assessment on a selected CBCT sagittal cut (showing the root 
tip of the impacted canine) by measuring the length of a perpendicular line drawn from the root tip to the long axis of the alveolar process and a score from 0 to 5 on the same KPG index basics was given (figure
2). The same approach was also used to assess the cusp tip when the maxillary canine was horizontally impacted as the first part of the tooth shown on the axial cut was not the cusp tip.

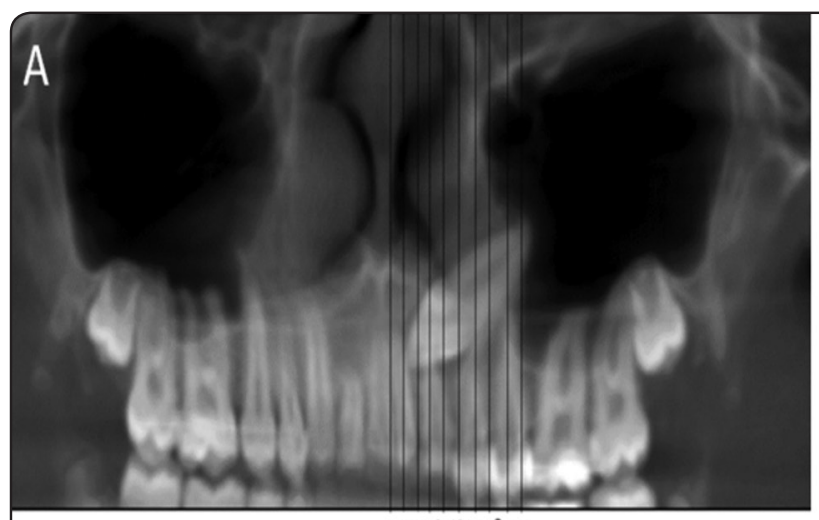

54321012345
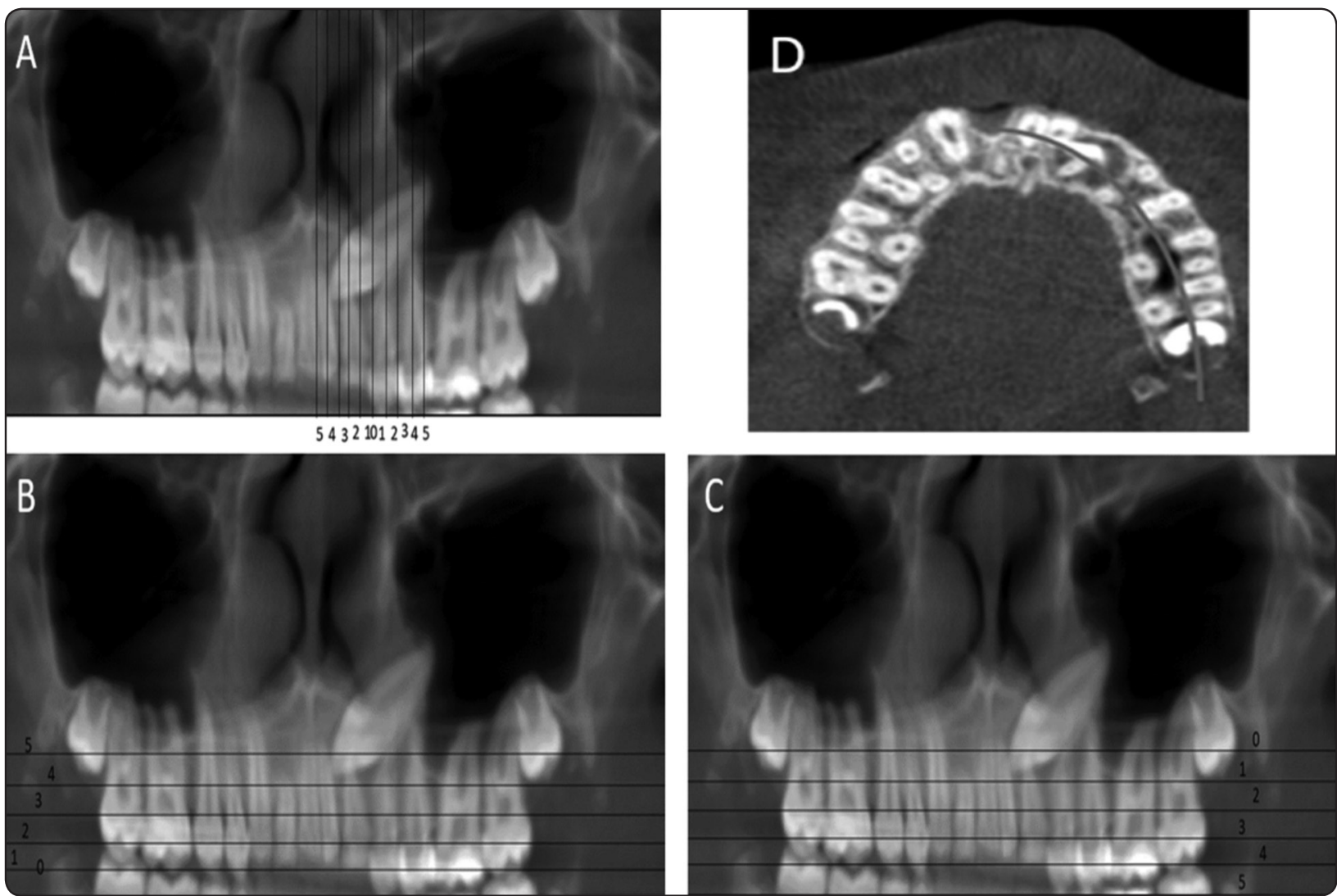

Fig. (1): KPG index applied on a case of 17 years female with an impacted left maxillary canine: (A) CBCT reformatted panoramic cut showing the mesio-distal (X axis) positions of both the cusp and root tips (scores $4 \& 5$ respectively) (B) CBCT reformatted panoramic cut showing the vertical (Y axis) position of the cusp tip (score 4) (C) CBCT reformatted panoramic cut showing the vertical (Y axis) position of the root tip (score 0) (D) CBCT axial cut showing the labio-lingual ( $\mathrm{Z}$ axis) position of the cusp tip (score 0 )in relation to the occlusal reference line

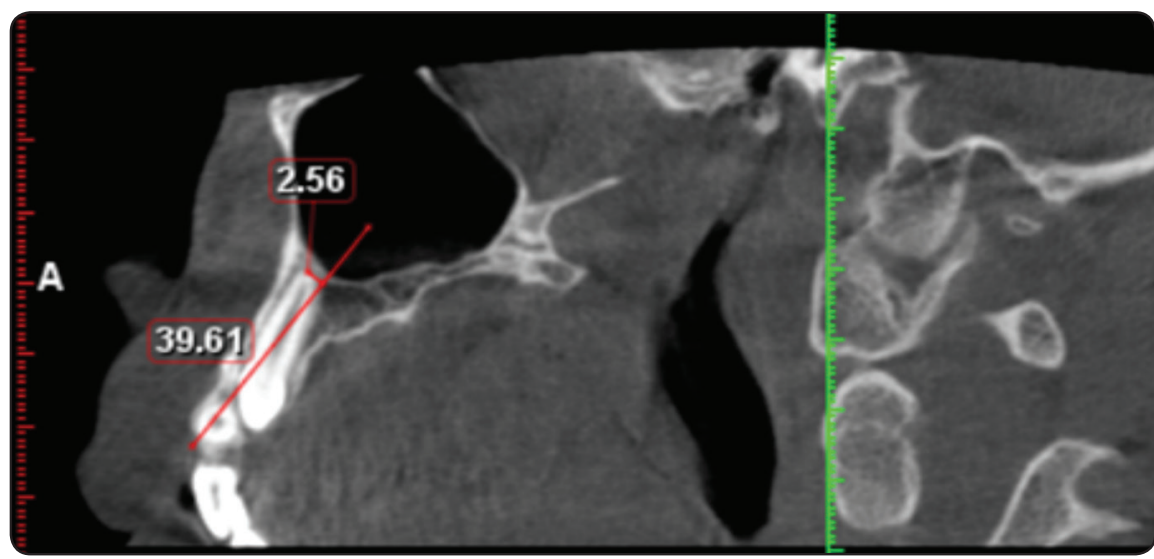

Fig. (2): Labio-lingual ( $\mathrm{Z}$ axis) localization of the impacted canine root tip on a selected CBCT sagittal cut by measuring the length of a perpendicular line connecting the root tip with the long axis of the alveolar process $(2.56 \mathrm{~mm}$ taking a score of 2) 


\section{Method applying the guidelines for the assessment of the impacted maxillary canine proposed by Counihan et al at $2013^{10}$ :}

On the same previously constructed CBCT reformatted panoramic cut, according to these guidelines, four aspects of the impacted maxillary canine alignment were assessed ${ }^{10}$ :

- The horizontal overlap of the canine crown on the adjacent incisor: good prognosis was given to the absence of overlap, average prognosis to the overlap up to half root width and poor prognosis was associated with the complete overlap of incisors.

- The vertical height of the canine crown: good prognosis was assigned to the impacted cusp tip when located vertically from the cementoenamel junction up to half the root length of the adjacent incisor, average prognosis when located at more than half but less than the full root length and poor prognosis when located above the entire root length.

- The canine angulation: the angle between the long axis of the impacted canine and the midline was plotted and measured where $0-15$ was considered good, 16-30 average and more than 30 degrees was given a poor prognosis.

- The position of the canine root apex in the horizontal plane: If its apex was located above the normal canine position, prognosis for alignment was considered good, if above the first premolar region, prognosis was average, and above the second premolar, prognosis was assumed as poor.

In order to compare the KPG index with the guidelines proposed by Counihan et al at 2013 (with only 3 grades), these four KPG categories were reduced to three, by just considering the difficult category with a score more than 15 .

\section{Statistical Analysis}

Data were collected, revised, coded and entered to the Statistical Package for Social Science (IBM SPSS) version 23. Data were presented as mean and standard deviations for quantitative data and numbers and percentages for qualitative data. The comparison between the two methods was done using Chi-square test for qualitative data. The confidence interval was set to $95 \%$ and the margin of error accepted was set to $5 \%$. So, the p-value was considered significant at the level of $<0.05$.

\section{RESULTS}

This study was conducted on 45 impacted maxillary canines found in 36 patients: 23 on the right $(51 \%)$ and 22 on the left (48.9\%) side: 6 males $(16.7 \%)$ and 30 females (83.3\%). Their age range was 13-35 years (mean age 21.61 \pm 5.97 years). 27 patients (75\% of cases) had unilateral impacted maxillary canines while 18 impactions were found bilaterally in 9 patients ( $25 \%$ of cases).

Upon comparing both methods (table1), a statistically significant difference was found. KPG index categorized more cases ( 9 cases) as easy (20\%), while Counihan et al guidelines categorized only 2 cases $(4.4 \%)$ as having good prognosis. Similarly, KPG index categorized more cases (15 cases) as moderate (33.3\%), while Counihan et al guidelines categorized 13 cases $(28.9 \%)$ as having average prognosis. On the opposite side, Counihan et al guidelines categorized more cases (30 cases) as poor prognosis $(66.7 \%)$, while KPG index categorized only 21 cases $(46.7 \%)$ as being difficult.

On comparing the treatment difficulty and prognosis of impacted maxillary canines found on the right and left sides (table 2), a non-statistically significant difference was found using both methods of radiographic assessment.

On comparing the treatment difficulty and prognosis of impacted maxillary canines found in males and females (table 3), a non-statistically significant difference was found using both methods of radiographic assessment.

On comparing the treatment difficulty and prognosis of impacted maxillary canines found either unilaterally or bilaterally (table 4), a nonstatistically significant difference was found using both methods of radiographic assessment. 
TABLE (1): Chi-square test comparing both methods of radiographic assessment of impacted maxillary canine:

\begin{tabular}{|c|c|c|c|c|c|}
\hline & KPG Index & Counihan et al Guidelines & \multirow[t]{2}{*}{ Test value } & \multirow[t]{2}{*}{ P-value } & \multirow[t]{2}{*}{ Sig. } \\
\hline & No. $=45$ & No. $=45$ & & & \\
\hline Easy (good) & $9(20.0 \%)$ & $2(4.4 \%)$ & \multirow[t]{3}{*}{6.186} & \multirow[t]{3}{*}{0.045} & \multirow[t]{3}{*}{ S } \\
\hline Moderate (average) & $15(33.3 \%)$ & $13(28.9 \%)$ & & & \\
\hline Difficult (poor) & $21(46.7 \%)$ & $30(66.7 \%)$ & & & \\
\hline
\end{tabular}

TABLE (2): Chi-square test comparing the right and left maxillary impacted canines using both methods of radiographic assessment:

\begin{tabular}{|c|c|c|c|c|c|c|}
\hline & & Right & Left & \multirow{2}{*}{ Test value } & \multirow{2}{*}{$\mathrm{P}$-value } & \multirow{2}{*}{ Sig. } \\
\hline & & No. $=23$ & No. $=22$ & & & \\
\hline \multirow{3}{*}{ KPG Index } & Easy & $4(17.4 \%)$ & $5(22.7 \%)$ & \multirow{3}{*}{2.185} & \multirow{3}{*}{0.335} & \multirow{3}{*}{ NS } \\
\hline & Moderate & $10(43.5 \%)$ & $5(22.7 \%)$ & & & \\
\hline & Difficult & $9(39.1 \%)$ & $12(54.5 \%)$ & & & \\
\hline \multirow{3}{*}{ Counihan et al Guidelines } & Good & $1(4.3 \%)$ & $1(4.5 \%)$ & \multirow{3}{*}{0.055} & \multirow{3}{*}{0.973} & \multirow{3}{*}{ NS } \\
\hline & Average & $7(30.4 \%)$ & $6(27.3 \%)$ & & & \\
\hline & Poor & $15(65.2 \%)$ & $15(68.2 \%)$ & & & \\
\hline
\end{tabular}

TABLE (3): Chi-square test comparing the maxillary impacted canines found in males and females using both methods of radiographic assessment:

\begin{tabular}{|c|c|c|c|c|c|c|}
\hline & & Females & Males & \multirow{2}{*}{ Test value } & \multirow{2}{*}{ P-value } & \multirow{2}{*}{ Sig. } \\
\hline & & No. $=45$ & No. $=45$ & & & \\
\hline \multirow{3}{*}{ KPG Index } & Easy & $7(18.9 \%)$ & $2(25.0 \%)$ & \multirow{3}{*}{0.347} & \multirow{3}{*}{0.841} & \multirow{3}{*}{ NS } \\
\hline & Moderate & $12(32.4 \%)$ & $3(37.5 \%)$ & & & \\
\hline & Difficult & $18(48.6 \%)$ & $3(37.5 \%)$ & & & \\
\hline \multirow{3}{*}{ Counihan et al Guidelines } & Good & $2(5.4 \%)$ & $0(0.0 \%)$ & \multirow{3}{*}{0.708} & \multirow{3}{*}{0.702} & \multirow{3}{*}{ NS } \\
\hline & Average & $10(27.0 \%)$ & $3(37.5 \%)$ & & & \\
\hline & Poor & $25(67.6 \%)$ & $5(62.5 \%)$ & & & \\
\hline
\end{tabular}

TABLE (4): Chi-square test comparing the maxillary impacted canines found unilaterally or bilaterally using both methods of radiographic assessment.

\begin{tabular}{|c|c|c|c|c|c|c|}
\hline & Unilateral & Bilateral & \multirow{2}{*}{ Test value } & \multirow{2}{*}{ P-value } & \multirow{2}{*}{ Sig. } \\
\hline & & 27 teeth & 18 teeth & & & \\
\hline \multirow{3}{*}{ KPG Index } & Easy & $4(14.8 \%)$ & $5(27.8 \%)$ & \multirow{3}{*}{4.312} & \multirow{3}{*}{0.116} & \multirow{3}{*}{ NS } \\
\hline & Moderate & $7(25.9 \%)$ & $8(44.4 \%)$ & & & \\
\hline & Difficult & $16(59.3 \%)$ & $5(27.8 \%)$ & & & \\
\hline \multirow{3}{*}{ Counihan et al Guidelines } & Good & $1(3.7 \%)$ & $1(5.6 \%)$ & \multirow{3}{*}{1.677} & \multirow{3}{*}{0.432} & \multirow{3}{*}{ NS } \\
\hline & Average & $6(22.2 \%)$ & $7(38.9 \%)$ & & & \\
\hline & Poor & $20(74.1 \%)$ & $10(55.6 \%)$ & & & \\
\hline
\end{tabular}




\section{DISCUSSION}

CBCT has been widely used for diagnosis of teeth impactions, especially impacted maxillary canines, as it allows three-dimensional visualization, precise localization, determination of amount of covering bone and assessment of their relation to adjacent anatomical structures ${ }^{2,17,18}$.

The KPG index represents a simplified method to assess the alignment and to formulate a rapid treatment difficulty score for impacted maxillary canines using CBCTs with no need for multiple linear and angular measurements ${ }^{4,16}$. Similarly, the four category guidelines suggested by Counihan et al at 2013 were proposed to aid in decisionmaking regarding the management of impacted maxillary canine cases ${ }^{10}$. Although treatment plan of impacted maxillary canines usually requires a good cooperation between different dental specialties including an orthodontist, an oral surgeon, a periodontist and an oral radiologist; most of the previous studies were directed to investigate the methods of the impacted maxillary canine assessment only by orthodontists ${ }^{19,20}$. Therefore, the first aim of our study was to compare and evaluate the guidelines proposed by Counihan et al 2013 and the KPG method for assessment of maxillary impacted canine position using CBCT images but from a radiographic point of view.

In our study, a higher incidence of impacted maxillary canines was found in females $(83.3 \%)$ as compared to males $(16.7 \%)$. This was in concordance with many previous studies that showed that these impactions are more commonly found in female patients than in males with a $2: 1$ ratio $^{4,6,15,17,21-}$ 23. A typically similar result to ours was found by Zarch et al at 2013 where the majority of their patients were females ( $80 \%)$. They explained that this may be due to the variances in the craniofacial growth and development influences between males and females or even due to the higher demand of females for aesthetic purposes resulting in more regular orthodontic visits compared to males ${ }^{22}$. Additionally, Queck et al 2003 suggested that the gender differences may be caused by the fact that the skull and jaw sizes are smaller in women than in men and a proper jaw size is an important factor that helps the development and eruption of teeth in exact order and place ${ }^{24}$. However, other studies as Pico et al 2017 found the number of male and female patients in a ratio of $1: 1$ and justified that by their reduced sample size (only 28 impacted canines in 20 patients $)^{25}$.

An almost equal distribution of impacted maxillary canines was found in our results on both sides (23 on the right $(51 \%)$ and 22 on the left (48.9\%) side). Nevertheless, Al Zoubi et al at 2017 reported a higher prevalence of impacted maxillary canines on the left side compared to the right side ${ }^{23}$. Similarly, a previous study by Takahama and Aiyama at 1982 reported a higher frequency of left sided maxillary impactions in cleft patients as well as in the control group. Both studies concluded that there are no scientific evidences to clarify the higher prevalence of left sided maxillary canine impactions and it might be only considered as a general feature of the malformation ${ }^{26}$.

In the current study, $75 \%$ of maxillary impacted canine cases were unilateral while only $25 \%$ were found bilaterally. Similarly, Pico et al 2017 found that the frequency of unilateral and bilateral impactions was $60 \%$ and $40 \%$ respectively ${ }^{25}$. Moreover, another previous study showed that the majority of impactions of maxillary canines are unilateral at $92 \%$, and only $8 \%$ are bilateral ${ }^{27}$.

Upon comparing both studied methods of radiographic assessment of impacted maxillary canines, a statistically significant difference was found: KPG index categorized more cases (9 cases) as easy (20\%) while Counihan et al guidelines categorized only 2 cases (4.4\%) as having good prognosis. Similarly, KPG index categorized more cases (15 cases) as moderate $(33.3 \%)$ while 
Counihan et al guidelines categorized 13 cases (28.9\%) as having average prognosis. On the other hand, Counihan et al guidelines categorized more cases (30 cases) as poor prognosis $(66.7 \%)$ while KPG index categorized only 21 cases $(46.7 \%)$ as being difficult.

This may indicate that Counihan et al guidelines categorized more cases as poor (difficult) prognosis and less cases as good (easy) compared to the KPG index method, revealing an overestimation of the poor category and an underestimation of the good prognosis. This may be explained on the basis that the considered guidelines categorized the impacted canines' position regarding their cusp tip position horizontally and vertically and the root tip horizontally only, simulating only three factors of the six factors considered in the KPG index. Moreover, these guidelines completely ignored the labio-lingual cusp or root orientation of the impaction, and didn't make use of the CBCT threedimensional volumetric data, which may result, in our opinion in some conflicts regarding their results.

On comparing the treatment difficulty and prognosis of impacted maxillary canines found on the right and left sides, a non-statistically significant difference was found using both methods of radiographic assessment. This may indicate that the side has no effect on the position of the impacted canines and accordingly their treatment duration or difficulty. Up to our knowledge, no other studies investigated the effect of the impacted tooth side on the case prognosis except a single study performed by Kim et al 2012 that focused on finding the properties of impacted canines, which affect its root resorption probability and found that the gender and distinction between right and left tooth positioning showed no significant difference and has no effect on their ability to resorb roots of adjacent teeth ${ }^{28}$.

On comparing the treatment difficulty and prognosis of impacted maxillary canines found in males and female, a non-statistically significant difference was found using both methods of radiographic assessment. This may minimize the effect of the patient's sex on the maxillary canine position and subsequently the faced treatment struggles. However, Al Hammadi et al 2018 studied the treatment difficulty index of maxillary canine impaction in Saudi population on panoramic radiographs and found that although, the canine impaction was twice as common in females as in males, the orthodontic treatment difficulty index was significantly higher in males than females ${ }^{29}$. This variation in their results than ours may be attributed to the difference in the followed methodology and studied population.

On comparing the treatment difficulty and prognosis of impacted maxillary canines found either unilaterally or bilaterally, a non-statistically significant difference was found using both methods of radiographic assessment. Again, this may demonstrate that the location of the impaction whether on one or both sides has no marked effect on the impacted tooth position and hence, its treatment scenario should be planned individually.

For the guidelines proposed by Counihan et al at 2013, the authors found them inadequate and even confusing for complete evaluation of the various probabilities of canine alignment. They suggested a more applicable rule for evaluating the final prognosis of the impaction by considering the major prognosis of the four assessed aspects and if equal (2 to 2) to take the worst prognosis. Additionally, some impacted canines couldn't be assessed following these guidelines such as those with their horizontal position of apex located above the incisors or the molars or those horizontally overlapping the central and not the lateral incisor.

For the KPG index, all our cases were scored 0 regarding the vertical position of the root tip that was situated at a much higher position than the estimated categories. Furthermore, the authors recommend the use of a properly selected sagittal cut for both 
the cusp and root tips instead of the axial cut applied in various previous studies ${ }^{15,16,30,31}$ as the proposed method is much more accurate particularly in different impaction positions. At the same direction, Uday et al 2014 concluded that the CBCT axial view was much reliable compared to sagittal and other views specifying that this depends mainly on the type of impaction. They also, recommended that the analysis using different CBCT views may lead the surgeon to a better surgical approach ${ }^{32}$.

To conclude, from a radiographic point of view, the KPG index, with some minute modifications, has demonstrated to be an effective and competent method for classification of maxillary impacted canine's treatment difficulty in accordance to their position, as compared to the guidelines proposed by

\section{Counihan et al at 2013.}

Unquestionably, in our opinion, the retrospective design of most of the studies, which focused on the correlation between the impacted canine position with treatment duration and difficulty, using KPG or any other strategy, contributed to weaken their clinical significance. The data depended more on a comparison of the indices with only the orthodontist point of view and treatment expectations based on theirexperience. They definitely lack the contribution of other dental specialties as radiologists, surgeons and even general practitioners, as well as the comparison with a more objective gold standard which is the actual treatment duration and protocol applied for each case with all faced difficulties. These recommendations were approved by other previous studies ${ }^{30,31,33}$.

A properly designed prospective clinical trial, taking into consideration these weak points, will be able to find a stronger evidence regarding factors influencing impacted maxillary canine's treatment duration and difficulty, allowing us also to clinically validate the KPG index or, even adjust it until reaching a new simple but reliable $3 \mathrm{D}$ index for impacted canine localization.

\section{REFERENCES}

1- Preda L, La FianzaA, Di Maggio EM, et al. The use of spiral computed tomography in the localization of impacted maxillary canines. Dentomaxillofac Radiol 1997; 26: 236-241.

2- Liu DG, Zhang WL, Zhang ZY, et al. Localization of impacted maxillary canines and observation of adjacent incisor resorption with cone-beam computed tomography. Oral Surg Oral Med Oral Pathol Oral Radiol Endod 2008; 105(1): 91-98.

3- Bedoya MM, Park JH. A Review of the Diagnosis and Management of Impacted Maxillary Canines. J Am Dent Assoc 2009; 140:1485-93.

4- Kau CH, Pan P, Gallerano RL, and English JD. "A novel 3D classification system for canine impactions - the KPG index," The International Journal of Medical Robotics and Computer Assisted Surgery 2009; 5(3): 291-296.

5- Sajnani AK, King NM. Complications associated with the occurrence and treatment of impacted maxillary canines. Singapore Dent J. 2014; 35:53-7.

6- Wriedt S, Jaklin J, Al-Nawas B, Wehrbein H. Unilateral impacted upper canines: examination and treatment proposal based on 3D versus $2 \mathrm{D}$ diagnosis. J Orofac Orthop. 2012; 73:28-40.

7- Alqerban A, Jacobs R, Fieuws S, Willems G. Comparison of two cone beam computed tomographic systems versus panoramic imaging for localization of impacted maxillary canines and detection of root resorption. Eur J Orthod 2011; 33: 93-102.

8- Bjerklin K, Ericson S. How a computerized tomography examination changed the treatment plans of 80 children with retained and ectopically positioned maxillary canines. Angle Orthod 2006; 76:43-51.

9- Hoseini Zarch SH, Heravi F, Javadian Langaroodi A, Pirgazi H. Evaluation of Cone Beam Computed Tomography in Diagnosis and Treatment Plan of Impacted Maxillary Canines. J Dent Mater Tech 2013; 2(3): 92-8.

10- Counihan K, Al-Awadhi EA, Butler J. Guidelines for the Assessment of the Impacted Maxillary Canine. Dent Update 2013; 40: 770-777.

11- McSherry P. The assessment of and treatment options for the buried maxillary canine. Dent Update 1996; 23(1): $7-10$. 
12- Stivaros N, Mandall NA. Radiographic factors affecting the management of impacted upper permanent canines. $\mathrm{J}$ Orthod 2000; 27(2):169-173.

13- Pitt S, Hamdan A, Rock P. A treatment difficulty index for unerupted maxillary canines. Eur J Orthod 2006; 28(2): $141-144$.

14- San Martin DE, English JD, Kau CH et al. "The KPG index - a novel 3D classification system for maxillary canine impactions," Texas Dental Journal 2012; 129: 265274.

15- Dalessandri D, Migliorati M, Rubiano R, Visconti L, Contardo L, Di Lenarda R, and Martin C. Reliability of a Novel CBCT-Based 3D Classification System for Maxillary Canine Impactions in Orthodontics: The KPG Index. The Scientific World Journal 2013; Article ID 921234:7 pages.

16- Kau CH, Lee JJ, Souccar NM. The validation of a novel index assessing canine impactions. Eur J Dent 2013;7: 399-404.

17- Haney E, Gansky SA, Lee JS, Johnson E, Maki K, Miller AJ, et al. Comparative analysis of traditional radiographs and cone-beam computed tomography volumetric images in the diagnosis and treatment planning of maxillary impacted canines. Am J Orthod Dentofacial Orthop 2010; 137:590-7.

18- Kau CH, Richmond S, Palomo JM, Hans MG. Three-dimensional cone beam computerized tomography in orthodontics. J Orthod 2005; 32:282-93.

19- Bishara SE. Clinical management of impacted maxillary canines. Semin Orthod 1998; 4:87-98.

20- Kokich VG. Surgical and orthodontic management of impacted maxillary canines. Am J Orthod Dentofacial Orthop 2004;126: 278-83.

21- Ericson S, Bjerklin K. The dental follicle in normally and ectopically erupting maxillary canines: a computed tomography study. Angle Orthod 2001;71: 333-42.

22- Zarch SHH, Heravi F, Langaroodi AJ and Pirgazi H. Evaluation of Cone Beam Computed Tomography in Diagnosis and Treatment Plan of Impacted Maxillary Canines. JDMT 2013; 2(3): 93-98.

23- Al-Zoubi H, Alharbi AA, Ferguson DJ, Zafar MS. Frequency of impacted teeth and categorization of impacted canines: A retrospective radiographic study using orthopantomograms. Eur J Dent 2017; 11:117-21.
24- Quek SL, Tay CK, Tay KH, Toh SL, Lim KC. Pattern of third molar impaction in a Singapore Chinese population: A retrospective radiographic survey. Int J Oral Maxillofac Surg 2003; 32:548-52.

25- Pico CLVR, do Vale FJF, Caramelo FJSFA, Corte-Real A, Pereira SMA. Comparative analysis of impacted upper canines: Panoramic radiograph Vs Cone Beam Computed Tomography. J Clin Exp Dent. 2017; 9 (10): e1176-82.

26- Takahama Y and Aiyama Y. Maxillary canine impaction as a possible microform of cleft lip and palate. Eur J Orthod $1982 ; 4: 275-7$.

27- Dachi SF, Howell FV. A survey of 3,874 routine full-mouth radiographs. II. A study of impacted teeth. Oral Surg Oral Med Oral Pathol 1961; 14(10):1165-1169.

28- Kim Y, Hyun HK and Jang KT. The position of maxillary canine impactions and the influenced factors to adjacent root resorption in the Korean population. European Journal of Orthodontics 34 (2012) 302-306.

29- Alhammadi MS, Asiri HA, Almashraqi AA. Incidence, severity and orthodontic treatment difficulty index of impacted canines in Saudi population. J Clin Exp Dent. 2018;10(4): e327-34.

30- Dalessandri D, Migliorati M, Visconti L, Contardo L, Kau $\mathrm{CH}$, and Martin C. KPG Index versus OPG measurements: A comparison between $3 \mathrm{D}$ and 2D methods in predicting teatment duration and difficulty level for patients with impacted maxillary canines. BioMed Research International 2014; Article ID 537620: 8 pages.

31- Olteanu C, Ganea I, Pop A, Lucaciu O, Tarmure V, Ionescu E, Teodorescu E, Chibelean M, Bud E, Pacurar M. CBCT evaluation of the severity of maxillary impacted canines using the KPG Index. REV.CHIM. 2018; 69 (6): 14581461 .

32- Uday NM, Kamath P, Kumar VA, Kumar AB, Scindhia R, Raghuraj MB, Rozario J. Comparison of axial and sagittal views for angulation, cuspal tip distance, and alveolus width in maxillary impacted canines using CBCT. J Orthod Res 2014; 2:21-6.

33- Sarıkır Ç, Alkurt MT, Değerli Ş, AltunkaynakB, Peker İ. Comparison of panoramic radiography and cone-beam computed tomography for qualitative and quantitative measurements regarding localization of permanent impacted maxillary canines. Acta Odontol Turc 2017; 34(1):1-7 